\title{
Response of Pulmonary Vein Potentials to Premature Stimulation
}

\author{
HIROSHI TADA, M.D., HAKAN ORAL, M.D., MEHMET OZAYDIN, M.D., \\ RADMIRA GREENSTEIN, M.D., FRANK PELOSI, JR., M.D., BRADLEY P. KNIGHT, M.D., \\ S. ADAM STRICKBERGER, M.D., and FRED MORADY, M.D.
}

From the Division of Cardiology, Department of Internal Medicine, University of Michigan, Ann Arbor, Michigan

\begin{abstract}
Pulmonary Vein Potentials. Introduction: Pulmonary vein potentials reflect depolarization of muscle fascicles within the myocardial sleeves that surround the pulmonary veins. The response of pulmonary vein potentials to premature stimulation has not been described.

Methods and Results: In 31 patients with paroxysmal atrial fibrillation referred for segmental isolation of the pulmonary veins, programmed stimulation with a single atrial extrastimulus was performed from the coronary sinus. Bipolar and unipolar electrograms were recorded with a decapolar Lasso catheter positioned sequentially within the left superior, left inferior, and right superior pulmonary veins, near the ostium. Premature stimulation often resulted in greater separation of the atrial and pulmonary vein potentials recorded within the pulmonary veins, fractionation of the pulmonary vein potentials, a change in the circumferential activation sequence of the pulmonary vein potentials, and dropout of pulmonary vein potentials.

Conclusion: In response to premature stimulation, the muscle fascicles within the myocardial sleeves that surround the pulmonary veins display a greater degree of decremental conduction than do the myocardial fibers in the adjacent left atrium, and they display heterogeneity in conduction properties and refractoriness. Discrimination of pulmonary vein potentials from atrial electrograms is facilitated by premature stimulation. (J Cardiovasc Electrophysiol, Vol. 13, pp. 33-37, January 2002)
\end{abstract}

pulmonary vein potential, myocardial sleeve, programmed stimulation

\section{Introduction}

Pulmonary vein potentials recorded circumferentially at the ostia of the pulmonary veins are a useful guide for segmental isolation of the pulmonary veins in patients with atrial fibrillation. ${ }^{1,2}$ Pulmonary vein potentials reflect depolarization in the muscle fascicles within the myocardial sleeves that surround the pulmonary veins. ${ }^{1,2}$ To gain insight into the electrophysiologic properties of the fascicles within the muscle sleeves, this study investigated the response of pulmonary vein potentials to premature stimulation.

\section{Methods}

\section{Patient Characteristics}

The subjects of this study were 31 patients with drugrefractory paroxysmal atrial fibrillation who underwent segmental isolation of the pulmonary veins. There were 26 men and 5 women (mean age $49 \pm 12$ years). Mean duration of symptomatic atrial fibrillation was $6.5 \pm 7.0$ years, and the patients experienced a mean of $14 \pm 11$ episodes per month. One patient had hypertension; the other 30 patients had no

Supported in part by a grant from the Gunma Prefecture Government and the Ellen and Robert Thompson Atrial Fibrillation Research Fund.

Address for correspondence: Fred Morady, M.D., Division of Cardiology, Department of Internal Medicine, University of Michigan, 1500 E. Medical Center Drive, B1F245, Ann Arbor, MI 48109-0022. Fax: 734-936-7026; E-mail: fmorady@umich.edu

Manuscript received 31 October 2001; Accepted for publication 27 November 2001. structural heart disease. Echocardiography demonstrated a mean left ventricular ejection fraction of $0.59 \pm 0.09$ (range 0.40 to 0.74 ), and a mean left atrial diameter of $38 \pm 5 \mathrm{~mm}$ (range 32 to 48 ).

\section{Electrophysiologic Study Protocol}

Therapy with antiarrhythmic drugs was discontinued at least 48 hours before the procedure. In the case of amiodarone, therapy was discontinued at least 6 weeks before the procedure. After informed consent was obtained, a quadripolar electrode catheter (EP Technologies Inc., San Jose, CA, USA) was inserted into the right femoral vein and positioned in the distal coronary sinus. Transseptal catheterization was performed and a 7-French decapolar electrode catheter with 1-mm electrodes spaced at intervals of 4.5 or $6.0 \mathrm{~mm}$ in a distal ring configuration (Lasso; Biosense Webster, Inc., Diamond Bar, CA, USA) was inserted into the left atrium. Heparin was administered as needed to maintain an activated clotting time of 250 to 350 seconds.

Surface leads and intracardiac electrograms were recorded digitally (EPMedSystems, Inc., Mount Arlington, NJ, USA). Filter settings were 30 to $500 \mathrm{~Hz}$ for bipolar electrograms and 0.05 to $200 \mathrm{~Hz}$ for unipolar electrograms. An indifferent electrode positioned on the right thigh was used for unipolar recordings. Programmed stimulation was performed with a stimulator (model EP-3 Clinical Stimulator; EPMedSystems) at twice diastolic threshold and pulse width of $2 \mathrm{msec}$.

\section{Study Protocol}

Pulmonary venous angiograms were performed to identify the location of the pulmonary vein ostia. The Lasso 


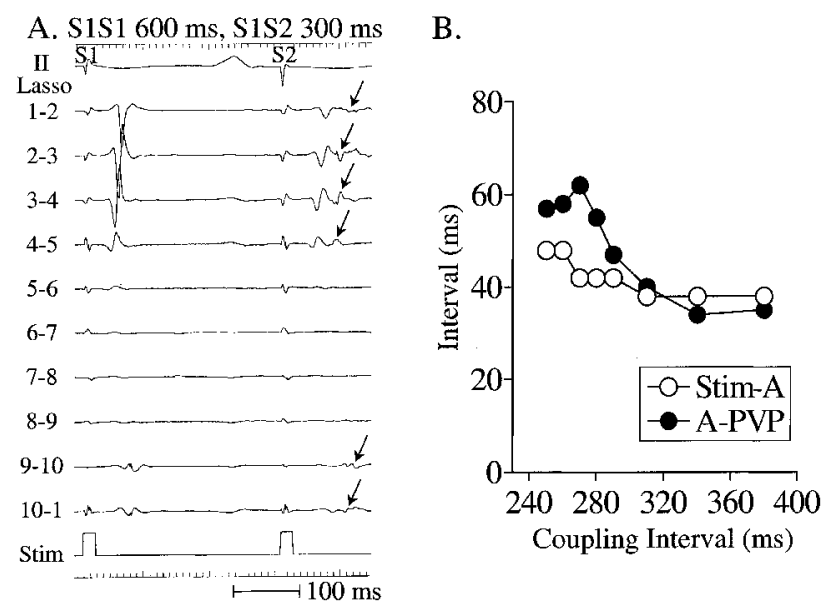

Figure 1. (A) Example of separation of atrial and pulmonary vein potentials during premature stimulation. Shown are lead II, 10 bipolar electrograms recorded by a Lasso catheter positioned in a left inferior pulmonary vein, and a stimulus (Stim) channel. During pacing (S1S1) at $600 \mathrm{~ms}$, there is overlap of the atrial and pulmonary vein potentials. At an S1S2 coupling interval of $300 \mathrm{~ms}$, the atrial and pulmonary vein potentials (arrows) became completely separated. (B) Representative example of the relationship between the S1S2 coupling interval and the stimulus-atrial (Stim-A) and atrial-pulmonary vein potential $(A-P V P)$ intervals.

catheter was positioned within the left superior pulmonary vein near the ostium, and programmed stimulation with one extrastimulus was performed from the coronary sinus at a basic drive cycle length of $600 \mathrm{msec}$. The initial S1S2 interval was 400 to $450 \mathrm{msec}$, and the S1S2 interval was shortened in steps of 10 to $20 \mathrm{msec}$ until atrial refractoriness was reached. Atrial fibrillation was never induced with this pacing protocol. Programmed stimulation was repeated after the Lasso catheter was positioned in the left inferior pulmonary vein, then again after it was positioned in the right superior pulmonary vein. The Lasso catheter usually could not be positioned within the right inferior pulmonary vein; therefore, electrograms recorded within this vein were not included in this study.

Bipolar electrograms were recorded with the 10 pairs of adjacent electrodes of the Lasso catheter, and unipolar electrograms were recorded with each of the 10 electrodes. Electrograms that were suitable for analysis in this study were obtained from 28 left superior pulmonary veins, 23 left inferior pulmonary veins, and 28 right superior pulmonary veins.

\section{Analysis of Electrograms}

Pulmonary vein potentials were defined as deflections that had a sharp upstroke, duration $<50 \mathrm{msec}$, amplitude $>0.05 \mathrm{mV}$, and that followed the atrial potential. ${ }^{1,3,4}$

Measurements of electrode width and timing were performed with electronic calipers. Analysis of bipolar electrograms consisted of the following measurements: the interval from the stimulus to the first deflection of the earliest atrial electrogram (Stim-A interval), and the interval from the first deflection of the earliest atrial electrogram to the first deflection of the earliest pulmonary vein potential (A-PVP interval). When there was overlap between the atrial and pulmonary vein potentials, the first deflection of the electrogram was taken as the onset of the atrial electrogram, and the second deflection of the electrogram was taken as the onset of the pulmonary vein potential. The electrograms generated during premature stimulation were compared with the electrograms generated during the basic drive train at a cycle length of $600 \mathrm{msec}$.

Fractionated pulmonary vein potentials were defined as pulmonary vein potentials that had multiple deflections in bipolar and unipolar recordings.

\section{Statistical Analysis}

Continuous variables are expressed as mean $\pm 1 \mathrm{SD}$ and compared using analysis of variance. When group differences were found, one-way analysis of variance was followed by the Tukey-Kramer method to test the significance of the difference among means in all groups. Categorical values were compared by Chi-square analysis. An overall Chi-square test for a $2 \times \mathrm{n}$ table was constructed, followed by a sequence of $2 \times 2$ Chi-square tests to compare the groups. $\mathrm{P}<0.05$ was considered statistically significant.

\section{Results}

\section{Stim-A and A-PVP Intervals}

Stim-A and A-PVP intervals both progressively lengthened as the S1S2 interval shortened (Figs. 1 and 2A).

The increase in A-PVP interval during premature stimulation resulted in less overlap between the atrial and pulmonary vein potentials recorded within the pulmonary veins. During pacing at a cycle length of $600 \mathrm{msec}$, the atrial and pulmonary vein potentials overlapped in $61 \%$ of left superior pulmonary veins, $47 \%$ of left inferior pulmonary veins, and $93 \%$ of right superior pulmonary veins. With premature stimulation, the percentage of pulmonary veins in which there was overlap of potentials decreased significantly to $12 \%$ to $54 \%$ in the three veins (Fig. 2B). As the S1S2 interval became progressively shorter, the A-PVP interval sometimes lengthened, then shortened (Fig. 3). This type of gap phenomenon was attributable to an increase in
A.

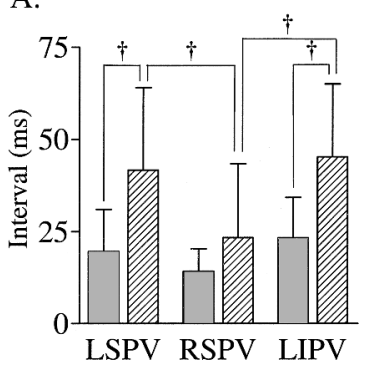

B.

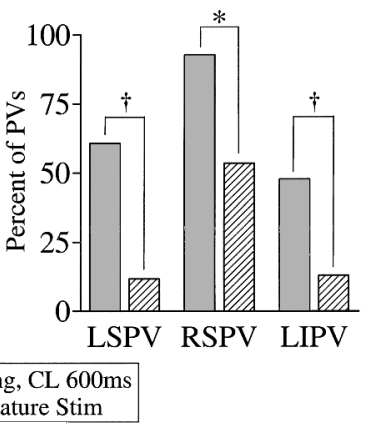

Figure 2. (A) Atrial-pulmonary vein potential intervals during pacing at a cycle length $(C L)$ of $600 \mathrm{~ms}$ and during premature stimulation. The maximum values observed during premature stimulation are shown. (B) Prevalence of overlap between atrial and pulmonary vein potentials recorded within pulmonary veins. Compared to pacing at $C L=600 \mathrm{~ms}$, the percentage of pulmonary veins in which bipolar electrograms demonstrated overlap of the atrial and pulmonary vein potentials significantly decreased during premature stimulation. $* P<0.05 ; \dagger P<0.001$. Error bars represent $1 S D$. LIPV = left inferior pulmonary vein; $L S P V=$ left superior pulmonary vein; $P V=$ pulmonary vein; $R S P V=$ right superior pulmonary vein. 
Figure 3. Example of a gap phenomenon in the atrialpulmonary vein potential $(A-P V P)$ interval during premature stimulation. Shown are lead II, 10 bipolar electrograms recorded by a Lasso catheter positioned in a left superior pulmonary vein, and a stimulus (Stim) channel. As the S1S2 interval shortened from 400 to $300 \mathrm{~ms}$, the A-PVP interval increased from 18 to $42 \mathrm{~ms}$. At an S1S2 of $250 \mathrm{~ms}$, the A-PVP interval shortened to $20 \mathrm{~ms}$, because of a marked lengthening of the stimulus-atrial (Stim-A) interval. With further shortening of the S1S2 interval to $230 \mathrm{~ms}$, the interval between the A-PVP interval again prolonged.

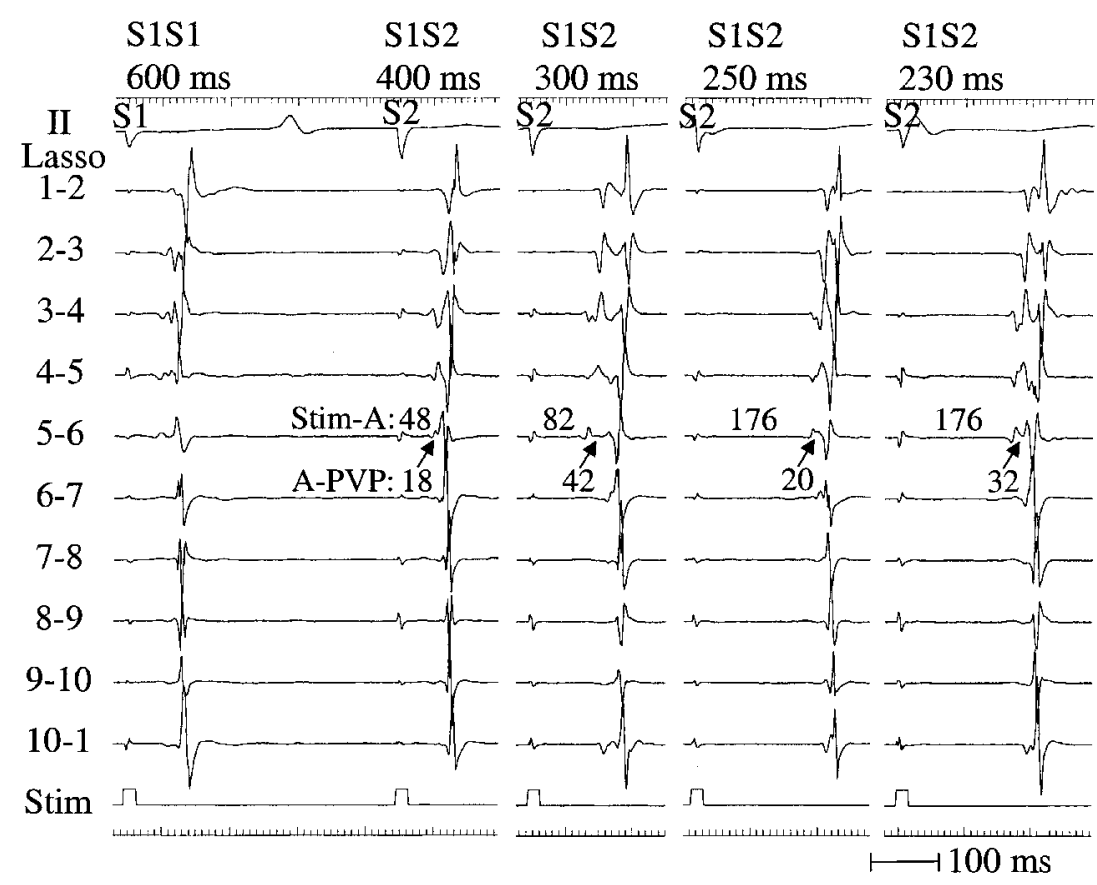

the A1A2 interval resulting from marked lengthening of the Stim-A interval and was observed in $29 \%$ of pulmonary veins.

\section{Fractionation of Pulmonary Vein Potentials}

Fractionated pulmonary vein potentials were not observed during pacing at a cycle length of $600 \mathrm{msec}$. During premature stimulation, fractionated pulmonary vein potentials were observed in $61 \%$ of pulmonary veins (Fig. 4).

\section{Changes in Activation Sequence and Conduction Block}

Compared to during pacing at a cycle length of 600 msec, during premature stimulation, the activation sequence of the circumferentially recorded pulmonary vein potentials changed in $32 \%$ of pulmonary veins (Fig. 5A).

During premature stimulation, conduction block in a pulmonary vein fascicle, manifest as dropout of a pulmonary vein potential, was observed in $15 \%$ of pulmonary veins (Fig. 5B).

\section{Discussion}

\section{Major Findings}

The results of this study demonstrate that the muscle fascicles that generate pulmonary vein potentials from within the myocardial sleeves that surround the pulmonary
Figure 4. Example of fractionation of a pulmonary vein potential during premature stimulation. Shown are lead II, 10 bipolar and unipolar electrograms recorded by a Lasso catheter positioned in a left inferior pulmonary vein, and a stimulus (Stim) channel. The last stimulus of the basic drive train (S1) results in overlap of the atrial and pulmonary vein potentials. Premature stimulation at a coupling interval of $320 \mathrm{~ms}$ results in fractionation of the pulmonary vein potentials, observed in both the bipolar and unipolar recordings (arrows).

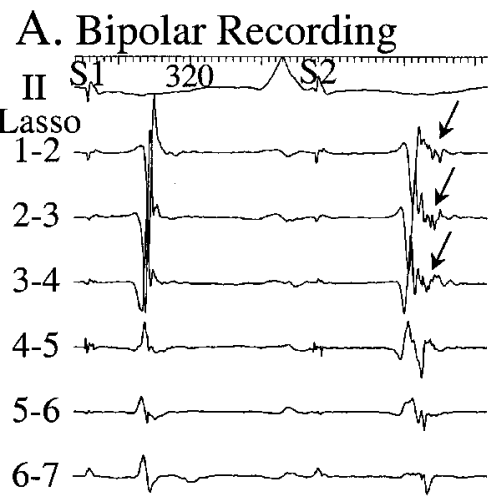

$7-8 \rightarrow r-r$

8-9

$9-10-2$

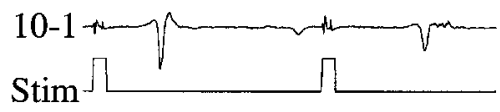

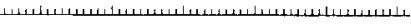

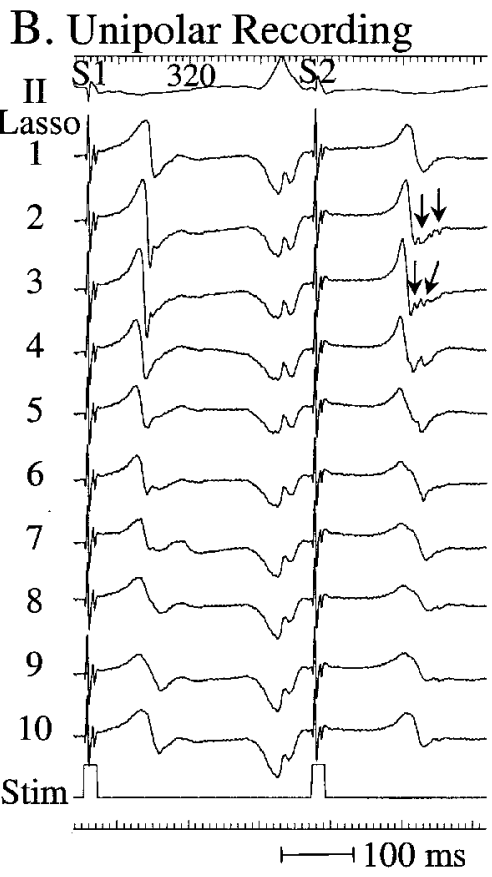



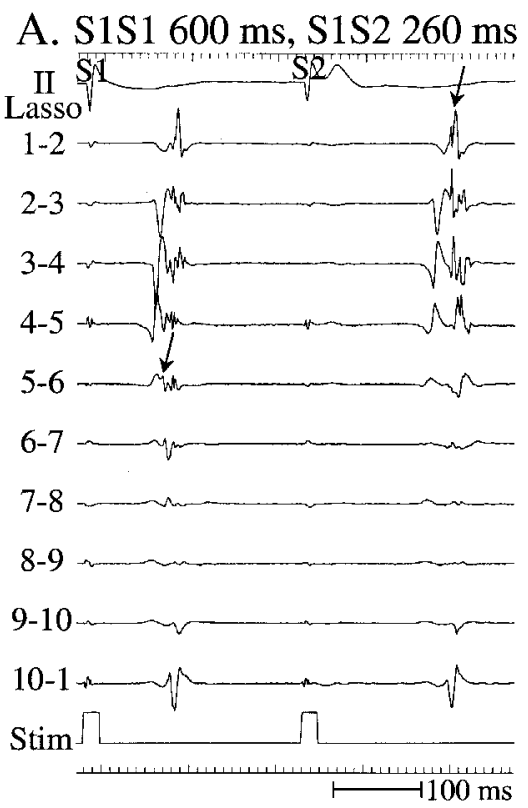

B. S1S1 $600 \mathrm{~ms}$, S1S2 $270 \mathrm{~ms}$

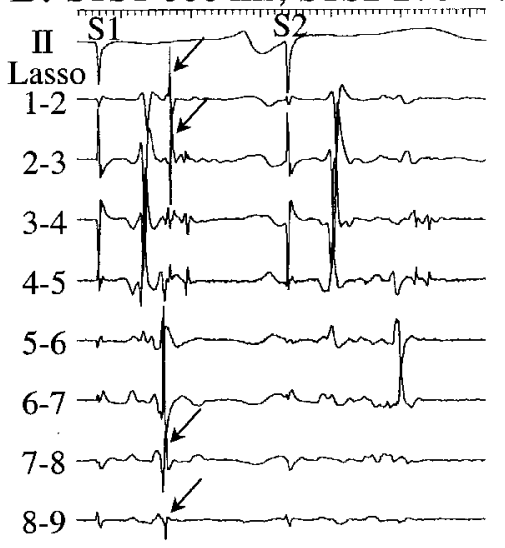

$9-10-10$

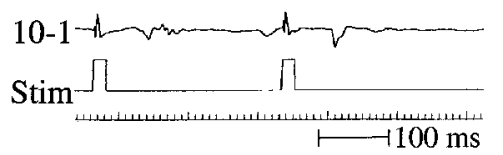

Figure 5. (A) Example of a change in activation sequence of the pulmonary vein potentials during premature stimulation. Shown are lead II, 10 bipolar electrograms recorded by a Lasso catheter positioned in a left superior pulmonary vein, and a stimulus (Stim) channel. With the last stimulus of the basic drive train (S1), there is partial overlap of the atrial and pulmonary vein potentials. The earliest pulmonary vein potential is recorded by electrodes 5-6 (arrow). Premature stimulation at a coupling interval of 260 ms resulted in a change in activation sequence of the pulmonary vein potentials, such that the earliest pulmonary vein potential was recorded by electrodes 1-2 (arrow). (B) Example of dropout of pulmonary vein potentials during premature stimulation. The last stimulus of the basic drive train (S1) and a premature stimulus (S2) at an S1S2 interval of 270 ms are shown. During pacing at a cycle length of $600 \mathrm{~ms}$, large pulmonary vein potentials are present and are separated from the atrial potentials. With premature stimulation, the pulmonary vein potentials recorded by electrodes 1-2, 2-3, 7-8, and 8-9 (arrows) are no longer present, and the intervals between the atrial electrograms and the remaining pulmonary vein potentials are prolonged.

veins display decremental conduction during premature stimulation. Decremental conduction also was observed in the left atrium, as manifest by a progressive increase in stimulus latency of the atrial electrograms recorded in the pulmonary veins during premature stimulation. However, the magnitude of slowing during premature stimulation was greater in the myocardial sleeves than in the left atrium, resulting in an increase in separation of the atrial and pulmonary vein potentials recorded in the pulmonary veins.

During premature stimulation, a change in the circumferential activation sequence of pulmonary vein potentials commonly was observed, as was complete dropout of pulmonary vein potentials. These observations demonstrate that the conduction properties and refractory periods of the muscle fascicles within the myocardial sleeves are heterogeneous.

\section{Electrophysiologic Characteristics of the Myocardial Sleeve}

The anatomic characteristics of the myocardial sleeves that surround the pulmonary veins recently have been described. ${ }^{5-7}$ However, little is known about the electrophysiologic characteristics of these myocardial sleeves. The myocardial sleeves are extensions of the atrial myocardium. They are composed of circumferential or spirally oriented bundles of myocytes that are not histologically specialized and that interconnect with each other and with other bundles that may have a longitudinal or oblique course, sometimes forming mesh-like arrangements. ${ }^{5,6}$ Fibrous tissue and adipose are interspersed among the myocardial fibers. ${ }^{6}$ It is possible that the complex arrangement of muscle fibers within the myocardial sleeves and the uneven distribution of interspersed connective tissue and adipose account for the greater degree of decremental conduction observed in the myocardial sleeves than in the left atrium and for the heterogeneity in conduction properties and refractory periods among the fascicles within the myocardial sleeves.

Fractionation of an electrogram usually indicates local slowing of conduction..$^{8-11}$ Therefore, the fractionation of pulmonary vein potentials commonly observed in this study during premature stimulation is consistent with anisotropic properties that may be attributable to the complex arrangement of muscle fascicles within the myocardial sleeves.

The mechanisms of tachycardias that originate in the pulmonary vein muscle sleeves have not been elucidated. The heterogeneity in conduction and refractoriness found in this study, along with the local slowing of conduction in response to premature stimulation, suggest that reentry may be one of the tachycardia mechanisms.

\section{Clinical Implications}

An endpoint of segmental isolation of the pulmonary veins is the elimination of all pulmonary vein potentials at the ostia of the pulmonary vein. ${ }^{1}$ Therefore, it is important to be able to distinguish atrial and pulmonary vein potentials recorded within the pulmonary veins and to be able to identify the circumferential activation sequence of the pulmonary vein potentials. This is facilitated by coronary sinus pacing at a cycle length of $600 \mathrm{msec}$, which often results in separation of the atrial and pulmonary vein potentials, at 
least in the left superior and inferior pulmonary veins. ${ }^{1,2,4}$ However, even during coronary sinus pacing at a cycle length of $600 \mathrm{msec}$, the atrial and pulmonary vein potentials still may overlap in approximately $50 \%$ to $60 \%$ of left pulmonary veins. ${ }^{4}$ The findings of this study demonstrate that when there is overlap of the electrograms, premature stimulation is useful in separating the pulmonary vein potentials from the atrial electrograms, facilitating the segmental isolation procedure.

\section{References}

1. Haissaguerre M, Shah DC, Jais P, Hocini M, Yamane T, Deisenhofer I, Chauvin M, Garrigue S, Clementy J: Electrophysiological breakthroughs from the left atrium to the pulmonary veins. Circulation 2000;102:2463-2465.

2. Hocini M, Shah DC, Jais P, Haissaguerre M, Peng JT, Yamane T, Deisenhofer I, Garrigue S, Clementy J: Concealed left pulmonary vein potentials unmasked by left atrial stimulation. PACE 2000;23(Pt II): 1832-1835.

3. Hsieh MH, Tai CT, Tsai CF, Yu WC, Lee SH, Lin YK, Ding YA, Chang MS, Chen SA: Pulmonary vein electrogram characteristics in patients with focal sources of paroxysmal atrial fibrillation. J Cardiovasc Electrophysiol 2000;11:953-959.

4. Tada H, Oral H, Greenstein R, Pelosi F Jr, Knight BP, Strickberger SA, Morady F: Differentiation of atrial and pulmonary vein potentials recorded circumferentially within pulmonary veins. J Cardiovasc Electrophysiol (Submitted).

5. Ho SY, Cabrera JA, Tran VH, Farre J, Anderson RH, SanchezQuintana D: Architecture of the pulmonary veins: Relevance to radiofrequency ablation. Heart 2001;86:265-270.

6. Saito T, Waki K, Becker A: Left atrial myocardial extensions onto pulmonary veins in humans: Anatomic observations relevant for atrial arrhythmias. J Cardiovasc Electrophysiol 2000;11:888-894.

7. Nathan H, Eliakim M: The junction between the left atrium and the pulmonary veins. An anatomic study of human hearts. Circulation 1966;34:412-422.

8. Spach MS: Microscopic basis of anisotropic propagation in the heart. In Zipes DP, Jalife J, eds: Cardiac Electrophysiology: From Cell to Bedside. Second Edition. WB Saunders, Philadelphia, 1995, pp. 204216.

9. de Bakker JMT, Hauer RNW, Simmers TA: Activation mapping: Unipolar versus bipolar recording. In Zipes DP, Jalife J, eds: Cardiac Electrophysiology: From Cell to Bedside. Second Edition. WB Saunders, Philadelphia, 1995, pp. 1068-1078.

10. de Bakker JMT, van Capelle FJL, Janse MJ, Tasseron S, Vermeulen JT, de Jonge N, Lahpor JR: Slow conduction in the infarcted human heart: "Zigzag" course of activation. Circulation 1993;88:915-926.

11. Stevenson WG, Weiss JN, Wiener I, Rivits SM, Nademanee K, Klitzner T, Yeatman L, Josephson M, Wohlgelernter D: Fractionated endocardial electrograms are associated with slow conduction in human: Evidence from pace-mapping. J Am Coll Cardiol 1989;13:369376. 